
\title{
28 Research Square \\ Primary Colonic Lymphoma: Report of Two Cases and a Literature Review
}

\section{Li Chen}

Zhejiang University School of Medicine Sir Run Run Shaw Hospital

\section{Engeng Chen}

Zhejiang University School of Medicine Sir Run Run Shaw Hospital

\section{Dongai Jin}

Zhejiang University School of Medicine Sir Run Run Shaw Hospital

zhangfa song ( $\square$ songzhangfa@zju.edu.cn )

Zhejiang University https://orcid.org/0000-0001-6301-1813

\section{Research Article}

Keywords: Case Report, Lymphoma, Colon

Posted Date: February 25th, 2021

DOl: https://doi.org/10.21203/rs.3.rs-242869/v1

License: (c) (i) This work is licensed under a Creative Commons Attribution 4.0 International License. Read Full License 


\section{Abstract}

Introduction: Primary colonic lymphoma is a highly rare malignant tumor. There is no standard treatment method for this disease. We report two cases of primary lymphoma of the colon successfully treated with chemotherapy and surgery respectively.

Case Presentation: In the first case, a 61-year-old female presented with the symptoms of abdominal pain for more than one month. The patient was diagnosed with a colonic tumor. However, immunohistochemical examinations confirmed the initial diagnosis of colonic lymphoma. The patient underwent laparoscopic assisted right hemicolectomy and followed by postoperative adjuvant chemotherapy with CHOP regimen (cyclophosphamide, doxorubicin, vincristine, and prednisone), combined with targeted therapy of rituximab. In the second case, a 78-year-old male presented with complaints abdominal distention for more than one year. Diffuse Large B-cell Lymphoma was definitely diagnosed by immunohistochemical examinations. And this patient treated by the systemic chemotherapy with $\mathrm{R}$ - $\mathrm{CHOP}$ regimen.

Conclusions: Primary colonic lymphoma is a rare type of $\mathrm{NHL}$. The clinical treatment is not standardized unlike many other types of lymphomas. Maybe it is mainly based on the symptoms to determine whether surgery or systemic chemotherapy. Rituximab is effective in some patients which may play an important role in the treatment of unresectable or asymptomatic colonic lymphoma.

\section{Background}

Primary colonic lymphoma is a rare malignant disease, although the gastrointestinal tract is the main site of extranodal NHL [1-2]. B-cell lymphoma is the third most common colorectal malignancy after colorectal cancer and neuroendocrine tumors, with an incidence of less than $0.5 \%$ [3-4]. Elderly patients account for the majority, according to previous statistics, but the etiology and standardized treatment is not well established. We report two cases of primary lymphoma of the colon successfully treated with chemotherapy and surgery respectively.

\section{Case Report}

\section{Case 1}

A 61-year-old Chinese female was admitted to the Sir Run Run Shaw Hospital, School of Medicine, Zhejiang University, Hangzhou, China, with abdominal pain for more than one month on September 22, 2017. There was no fever and no change in stool characteristics and stool habits. Except for the presence of pallor, the general physical examination was normal. Routine laboratory investigations were within normal limit except the hemoglobin of $8.9 \mathrm{~g} / \mathrm{dl}$ and Lactate dehydrogenase was higher than normal value. Colonoscopy showed an ascending colonic mass with partial luminal obliteration. Contrastenhanced computed tomography (CT) revealed a malignant mass $(4.9 \mathrm{~cm} \star 3.8 \mathrm{~cm})$ of ascending colon with peripheral lymph node metastasis, breakthrough serosa layer (Figure 1). The indices of tumor 
markers, including cancer antigen (CA)-125, CA19-9, and carcinoembryonic antigen, were within normal limits. After initial pathological examination, the mass was considered to be lymphoma.

Immunohistochemically, tumor cells were positive for cluster of differentiation (CD)20, CD10, and BCL-6, but negative for CD3 and MUM-1, Ki-67 was $80 \%$ (Figure 2). Finally, the clinical impression and results of the immunohistochemical examination established a final diagnosis of Diffuse Large B-cell Lymphoma (DLBCL). Considering that the patient had abdominal pain and incomplete intestinal obstruction, the patient underwent laparoscopic assisted right hemicolectomy on September 26, 2017. The operation process was successful and the patient recovered well after operation. Postoperative pathological examination confirmed the diagnosis of Diffuse Large B-cell Lymphoma.

After that, the patient was transferred to the Department of Hematology, and PET-CT revealed the enlarged cervical lymph nodes. The diagnosis was confirmed as DLBCL GCB type, stage IIIA, and international prognostic index (IPI) score was 4. After exclusion of chemotherapy contraindications, six cycles of R-CHOP chemotherapy were performed from November 2017 to March 2018. The chemotherapy regimen was rituximab, cyclophosphamide, vindesine, doxorubicin and prednisone. After three years of follow-up, no tumor recurrence was found.

\section{Case2}

A 78-year-old Chinese male was admitted to the Sir Run Run Shaw Hospital, School of Medicine, Zhejiang University, Hangzhou, China, with complaints abdominal distention and pain for more than one year on June 15,2018 . The pain was not very severe, but it had been increasing in recent months. There was no nausea and vomiting, no anal exhaust, no fever, no bloody stool and other discomfort. Results of laboratory parameters were normal except that hemoglobin was only $7.7 \mathrm{~g} / \mathrm{dl}$. Contrast-enhanced computed tomography (CT) revealed that the transverse colon wall was thickened, the lumen was not narrow, and multiple enlarged lymph nodes were found around the serosa layer (Figure 3). Colonoscopy showed irregular hyperplasia, congestion and crispness of the intestinal mucosa in the middle part of the transverse colon. After multipoint biopsy, malignant tumor was considered as pathological result. Immunohistochemically, tumor cells were positive for CD20 and BCL-6, but negative for CD3 and CD10, Ki-67 was $90 \%$. Finally, the patient's Diffuse Large B-cell Lymphoma was definitely diagnosed. As the patient has no obvious intestinal symptoms, he was transferred to the Department of Hematology. After exclusion of chemotherapy contraindications, six cycles of reduced dose R-CHOP chemotherapy were performed from July 2018 to November 2018. Not long ago, the patient came to the hospital for followup, CT and colonoscopy showed no recurrence of the tumor.

\section{Discussion}

Non-Hodgkin's lymphomas (NHLs) are a group of malignancies that originate from B-cell precursors, Tcell precursors, mature B-cells, mature T-cells, or natural killer cells (in a few cases). Aggressive lymphomas are usually acute or subacute, with manifestations of a rapidly growing mass, systemic $B$ symptoms (i.e., fever, night sweats, weight loss), and/or elevated serum lactate dehydrogenase and uric 
acid, and include diffuse large B-cell lymphoma, Burkitt's lymphoma, adult T-cell leukemia/lymphoma, and precursor B- and T-cell lymphoblastic leukemia/lymphoma [1-5]. Indolent lymphomas are usually more occult, with manifestations of slowly progressive lymphadenopathy, hepatomegaly, splenomegaly, or hypocytosis, and mainly include follicular lymphoma, chronic lymphocytic leukemia/small lymphocytic lymphoma, and splenic marginal zone lymphoma [4-6].

Gastrointestinal lymphomas account for $5-10 \%$ of all NHLs, most of which occur in the stomach (accounting for $68-75 \%$ of all gastrointestinal lymphomas), followed by the small intestine (15-20\%), with the rest occurring in the colon and rectum [1-4]. The gastrointestinal tract is the main site of extranodal NHL. Lymphoma can involve a single primary site and can also affect multiple gastrointestinal sites, and local and distant lymph nodes can also be involved. Primary gastrointestinal NHL is rare, accounting for only $1-4 \%$ of malignancies originating from the stomach, small intestine, or colon [7-11]. B-cell lymphoma is the third most common colorectal malignancy after colorectal cancer and neuroendocrine tumors, with an incidence of less than 0.5\% [5-7]. Diffuse Large B-cell Lymphoma (DLBCL) is the most common histological subtype of gastrointestinal lymphoma, which is more aggressive than other B-cell lymphoma [12-13]. As we reported in our two cases of colonic lymphoma, the final pathological results both were DLBCL.

The pathogenesis of gastrointestinal lymphomas is associated with a variety of factors, and no specific risk factors have been identified. Helicobacter pylori infection is closely associated with the development of gastric mucosa-associated lymphoid tissue (MALT) lymphoma and is also related to other gastrointestinal lymphomas with a slightly lower degree of association [14-15]. Many autoimmune diseases are associated with an increased risk of lymphoma, including rheumatoid arthritis, Sjogren's syndrome, systemic lupus erythematosus, and granulomatosis with polyangiitis, which may be due to the increased cancer risk with immunosuppressive therapy [16].

Colorectal B-cell lymphoma has a variety of clinical manifestations, and its symptoms depend on the locations of lesions. In the colon, the incidence of right colon is higher than other parts [17]. Our two cases of lymphoma were located in ascending colon and transverse colon respectively. The most common symptoms are abdominal pain, weight loss, abdominal mass, and blood in the stool, as well as nausea, vomiting, altered bowel habits, obstruction, and acute peritonitis due to intussusception and intestinal perforation [18]. Fan et al. found that abdominal pain and weight loss occurred in $62 \%$ and $43 \%$ of 37 patients, respectively, while Bairey et al. reported that abdominal pain and weight loss occurred in $56 \%$ and $29 \%$ of patients, respectively [3-4,19]. Complete intestinal obstruction is a very rare clinical manifestation because colorectal lymphoma is more malleable than adenocarcinoma and does not promote connective tissue hyperplasia; however, incomplete intestinal obstruction is more common. Similarly, the main symptoms were abdominal pain and anemia in out two cases. Surprisingly, in most of the colorectal B-cell lymphoma cases reported in the literature, B symptoms usually do not exist, and fever is a rare symptom, which may be due to the initial presence of intestine-related symptoms $[6,20]$. 
The most common imaging examination for colorectal lymphomas is contrast-enhanced abdominal computed tomography (CT). Abdominal CT mainly provides information on tumor size, invasion depth, and local lymph node metastasis. Notably, CT cannot distinguish adenocarcinoma and lymphoma very well, and the diagnosis must be confirmed by colonoscopic biopsy. Colonoscopic findings can include diffuse mucosal nodules, colitis-like changes associated with induration and ulcers, or masses with or without ulcers [16-18]. Multipoint biopsy should be performed for all lesions using standard methods, and tumor classification requires immunohistochemistry. Comprehensive examinations, including peripheral blood tests, biochemical tests, CT scans of the chest and abdomen, and bone marrow biopsy, are needed to exclude systemic involvement and identify disease stages. In our case report, both cases were diagnosed by multipoint biopsy combined with immunohistochemistry. The most widely used staging system in clinical practice is the Lugano classification, a modification of the Ann Arbor classification $[13,20]$.

The main treatment for colorectal lymphoma is combined therapy, including surgery and chemotherapy. Early-stage tumors are mainly treated with surgery combined with postoperative chemotherapy, and advanced tumors are treated with multidrug chemotherapy (CHOP protocol) [21-22]. However, due to the effectiveness of targeted drugs in recent years, especially rituximab (RTX), CD20-positive B-cell lymphoma can be treated with multidrug chemotherapy combined with immunotherapy, and the treatment efficacy is long-lasting and complete [23-24]. According to current case reports, immunotherapy can obviate surgery, which is mainly performed to relieve pain and for emergencies, such as intestinal obstruction, intestinal perforation, and hemorrhage [25-26]. The best treatment for rapidly proliferating and aggressive advanced colorectal lymphoma is chemotherapy. The CHOP chemotherapy regimen (cyclophosphamide, doxorubicin, vincristine, and prednisone) is the primary treatment for intermediatestage and advanced B-cell lymphomas. Adding RTX into the standard CHOP regimen may improve the progression-free survival rate and overall survival rate [21-24]. If the treatment response is incomplete or the probability of recurrence is high, bone marrow transplantation or stem cell transplantation is performed. Because the disease is rare, current guidelines still lack high-level evidence for the selection of the best treatment for gastrointestinal lymphomas.

Although the treatment has improved in recent years, primary colorectal lymphoma, especially DLBCL, is still an invasive disease with poor prognosis [27]. Fan et al. found that tumor stage was the most important prognostic factor for survival [4]. Other researchers have found that histological grade is the most important prognostic factor. The international prognostic index (IPI) is a commonly used prognostic scoring system for non-Hodgkin's lymphoma and is also useful for colorectal lymphoma.

In our first case, because of the obvious symptoms of abdominal pain, incomplete intestinal obstruction, we adopted the treatment of surgical resection and postoperative adjuvant chemotherapy. In the second case, there was no obvious discomfort, so systemic chemotherapy was used. The two patients were treated with CHOP regimen (cyclophosphamide, doxorubicin, vincristine, and prednisone), combined with targeted therapy of rituximab. Fortunately, the therapeutic effect was very effective, although the treatment method was different. 
The present study aimed to increase awareness among clinicians. Primary colonic lymphoma is a rare type of NHL. The clinical treatment is not standardized unlike many other types of lymphomas. Maybe it is mainly based on the symptoms to determine whether surgery or systemic chemotherapy. Rituximab is effective in some patients which may play an important role in the treatment of unresectable or asymptomatic colonic lymphoma [28]. Long-term efficacy needs further follow-ups and large-scale research in the future.

\section{References}

[1] Mouchli M A, Ouk L, Scheitel M R, et al. Colonoscopy surveillance for high risk polyps does not always prevent colorectal cancer. World J Gastroenterol. 2018;24(8):905-916

[2] Zinzani PL, Magagnoli M, Pagliani G, Bendandi M, Gherlinzoni F, Merla E, et al. Primary intestinal lymphoma: clinical and therapeutic features of 32 patients. Haematologica. 1997;82:305-8

[3] Tahir M, Samad K, Koenig T, Viswanathan P. A rare case of primary diffuse large B-cell lymphoma of the colon. AME Case Rep. 2018, 2:28

[4] Fan CW, Changchien CR, Wang JY, et al. Primary colorectal lymphoma. Dis Colon Rectum. 2000;43:1277-82

[5] Shaye OS, Levine AM. Marginal zone lymphoma. J Natl Compr Cancer Netw, 2006; 4: 311-18

[6] Quayle FJ, Lowney JK. Colorectal lymphoma. Clin Colon Rectal Surg. 2006, 19:49-53

[7] Yoshino T, Ichimura K, Mannami T et al. Multiple organ mucosa-associated lymphoid tissue lymphomas often involve the intestine. Cancer, 2001; 91: 346-53

[8] Ha CS, Cho MJ, Allen PK et al. Primary non-Hodgkin lymphoma of the small bowel. Radiology, 1999; 211: $183-87$

[9] Pandey M, Wadhwa MK, Patel DD, Kothari KC. Primary malignant large bowel lymphoma. Am Surg. 2002;68:121-6

[10] Ferreri AJ, Montalban C. Primary diffuse large B-cell lymphoma of the stomach. Crit Rev Oncol Hematol, 2007; 63: 65-71

[11] Zhai L, Zhao Y, Lin L, Tian Y, Chen X, Huang H, Lin T. Non-Hodgkin's lymphoma involving the ileocecal region: a single-institution analysis of 46 cases in a Chinese population. J Clin Gastroenterol. 2012;46(6):50914

[12] Busch E, Rodriguez-Bigas M, Mamounas E, Barcos M, Petrelli NJ. Primary colorectal non-Hodgkin's lymphoma. Ann Surg Oncol. 1994;1:222-8 
[13] She WH, Day W, Lau PY, Mak KL, Yip AW. Primary colorectal lymphoma: case series and literature review. Asian J Surg. 2011;34(3):111-4

[14] Matsumoto T, lida M, Shimizu M. Regression of mucosa-associated lymphoid-tissue lymphoma of rectum after eradication of Helicobacter pylori. Lancet, 1997; 350: 115-16

[15] Nakamura S, Yao T, Aoyagi K et al. Helicobacter pylori and primary gastric lymphoma. A histopathologic and immunohistochemical analysis of 237 patients. Cancer, 1997; 79(1): 3-11

[16] Zhou JL, Qiu HZ, Sun J, Li J. The diagnosis and treatment of colorectal non-Hodgkin's lymphoma 32 cases. Zhonghua Wai Ke Za Zhi. 2011;49(4):290-4

[17] She WH, Day W, Lau PY, Mak KL, Yip AW. Primary colorectal lymphoma: case series and literature review. Asian J Surg. 2011;34(3):111-4

[18] Tevlin R, Larkin JO, Hyland JM, O'Connell PR, Winter DC 2. Primary colorectal lymphoma - a single centre experience. Surgeon. 2015;13(3):151-155.

[19] Bairey O, Ruchlemer R, Shpilberg O. Non-Hodgkin's lymphomas of the colon. Isr Med Assoc J. 2006;8:832-5.

[20] Li B, Shi YK, He XH et al. Primary non-Hodgkin lymphomas in the small and large intestine: Clinicopathological characteristics and management of 40 patients. Int J Hematol, 2008; 87: 375-81

[21] Pfreundschuh M, Kuhnt E, Trümper L, et al. CHOP-like chemotherapy with or without rituximab in young patients with good-prognosis diffuse large-B-cell lymphoma: 6-year results of an open-label randomised study of the MabThera International Trial (MInT) Group. Lancet Oncol. 2011, 12:1013-1022

[22] Hammel P, Haioun C, Chaumette MT et al. Efficacy of single-agent chemotherapy in low-grade B-cell mucosa-associated lymphoid tissue lymphoma with prominent gastric expression. J Clin Oncol, 1995; 13: 2524-29

[23] Salar A, Domingo-Domenech E, Estany C, Canales MA, Gallardo F, Servitje O, et al. Combination therapy with rituximab and intravenous or oral fludarabine in the first-line, systemic treatment of patients with extranodal marginal zone B-cell lymphoma of the mucosa-associated lymphoid tissue type. Cancer. 2009;115:5210-7

[24] Raderer $M$, Jager $G$, Brugger $S$ et al. Rituximab for treatment of advanced extranodal marginal zone $B$ cell lymphoma of the mucosa-associated lymphoid tissue lymphoma. Oncology, 2003; 65: 306-10

[25] Koniaris LG, Drugas G, Katzman PJ, Salloum R. Management of gastrointestinal lymphoma. J Am Coll Surg, 2003; 197: 127-41 
[26] Cai S, Cannizzo F Jr, Bullard Dunn KM, Gibbs JF, CzuczmanM RA. The role of surgical intervention in non-Hodgkin's lymphoma of the colon and rectum. Am J Surg. 2007;193:409-12

[27] Freeman C, Berg JW, Cutler SJ. Occurrence and prognosis of extranodal lymphomas. Cancer, 1972; 29: $252-60$

[28] Morrison VA. Evolution of R-CHOP therapy for older patients with diffuse large B-cell lymphoma. Expert Rev Anticancer Ther. 2008;8:1651-8

\section{Figures}

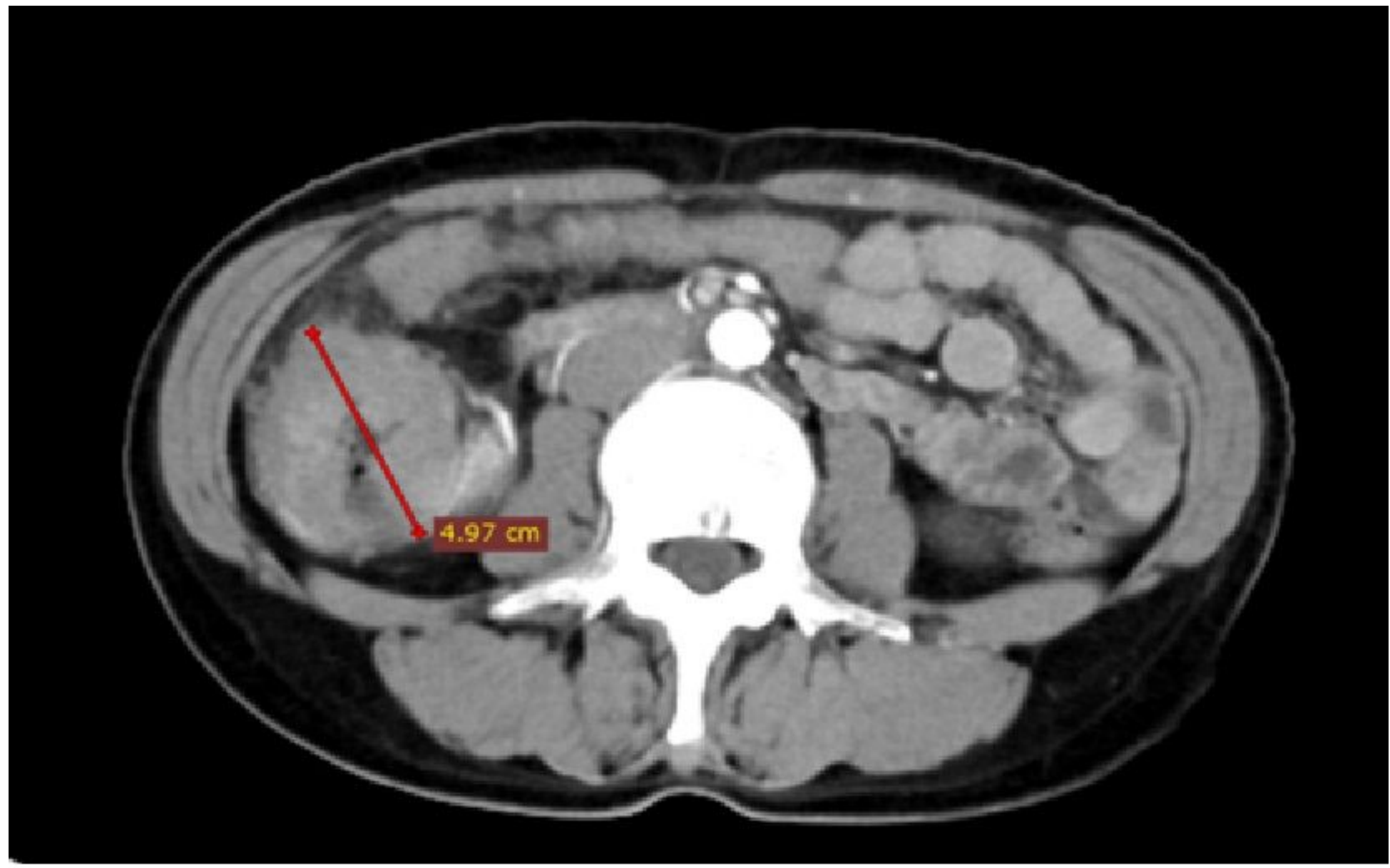

\section{Figure 1.}

\section{Figure 1}

Contrast-enhanced computed tomography (CT) of abdomen (horizontal view) showing an ascending colonic mass 

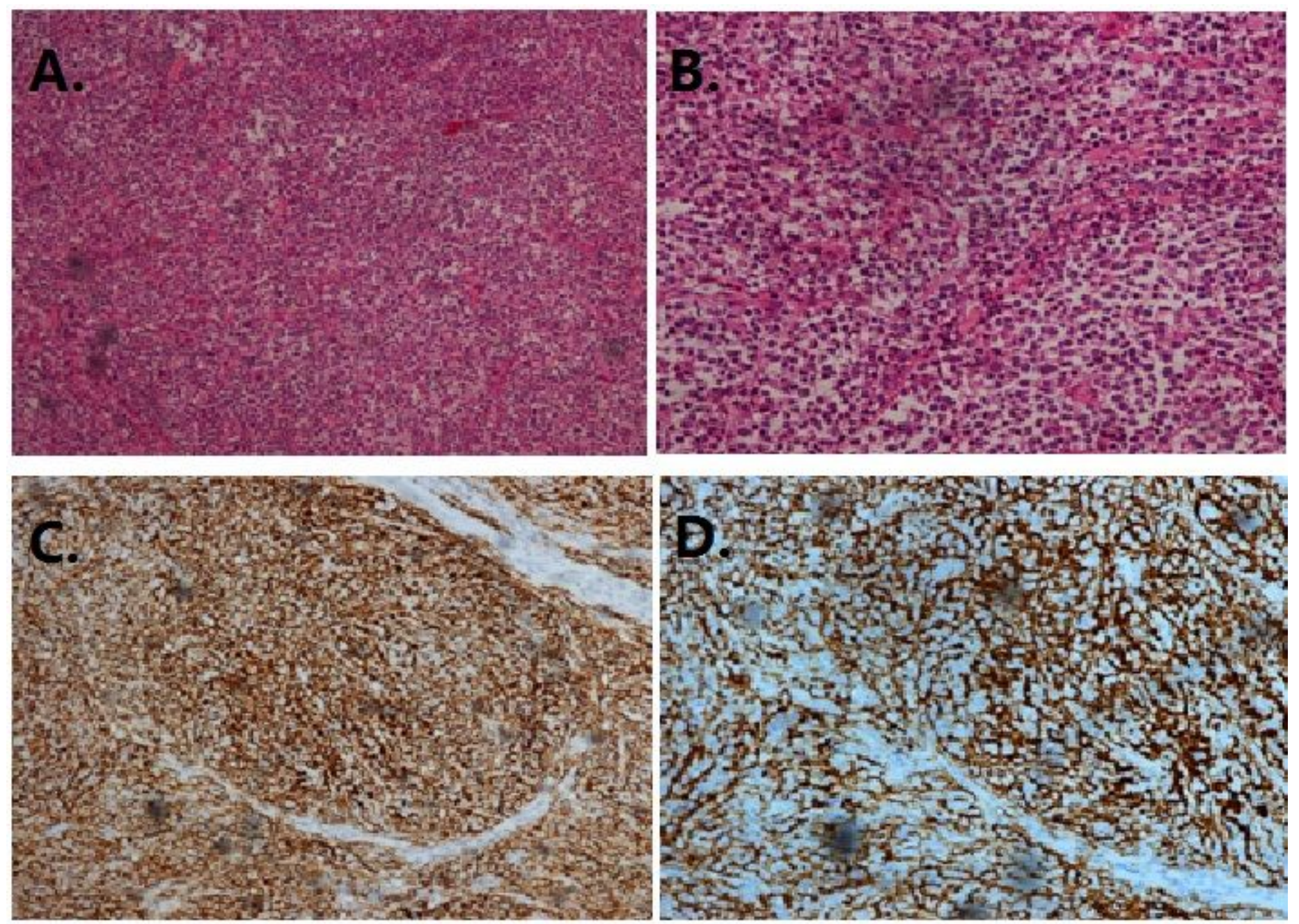

\section{Figure2.}

\section{Figure 2}

Histological features and immunohistochemical results of the colonic lymphoma. (A) The morphological examination (hematoxylin and eosin staining; magnification, x100). (B) The morphological examination (hematoxylin and eosin staining; magnification, x200). The tumor cells were demonstrated marked positivity for (C) CD10 and (D) CD20 (immunostaining; magnification, x200). CD, cluster of differentiation. 


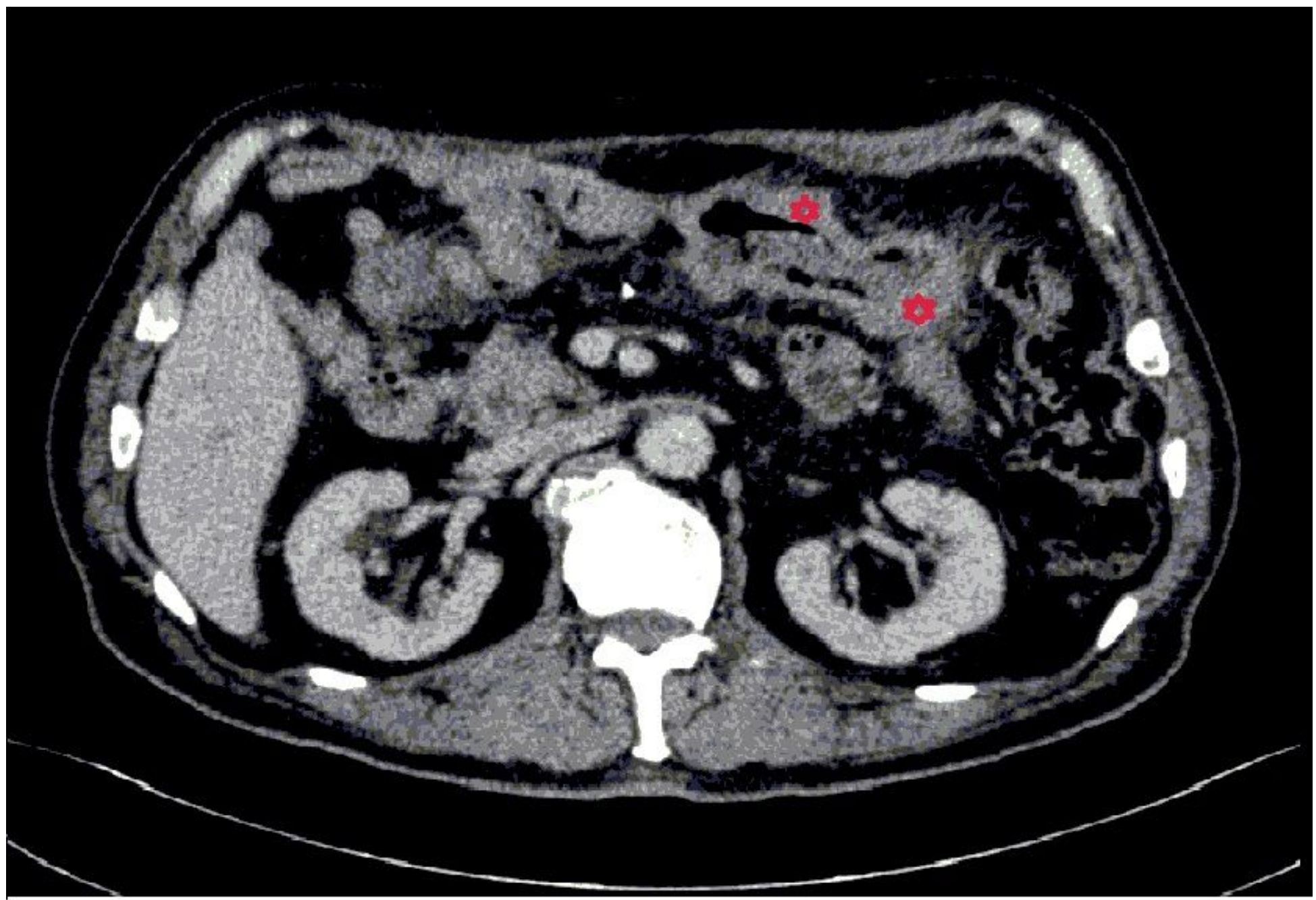

Figure 3.

Figure 3

Contrast-enhanced computed tomography (CT) of abdomen (horizontal view) showing the thickened colon wall (red star marked) 